hollow-ground scalpels, made by Messrs. Down Brothers to my design. The ordinary scalpel is quite unsuitable for skin division at any time unless extremely sharp. It is wedge-shaped and, may I say, the worst treated of all instruments. Is it not thrown into the tray, on the bed, and generally neglected? If an ordinary scalpel is used in my new skin operation nine out of ten cases will prove a failure. There must be one clean cut without force. The ordinary scalpel used as it should be will seldom do this, but more often dissect up a quarter of an inch or more epitbelial flap before penetrating the corium. My hollow-ground scalpel must be used only for the purpose for which it is intended and substituted for the ordinary scapel as soon as the skin is divided. It must be treated with the greatest care and after stropping cleansed with ether and alcohol. lisht spring tissue forceps, dissecting forceps, and sometimes lance-pointed needles and hurse-hair sutures are required, as also are dressings, glass cover, collodion, silk, absorbent wool, bandages, and sometimes a back splint.

With regard to the operation itself, the skin should be reasonably stretched in all directions by assistants if this is not already the case by some underlying tumour. The angle at which the knife should be held can only be learnt by practice. The skin, as a rule, will give five or six millimetres or about a quarter of an inch flap, but in addition at least as much tat flap, except, of course, when the $f$ at is excessive. Fat makes firm rapid union and is there. fore of considerable importance. The knife should make one clean sweep down to the fascia, which in turn should be divided as far under the base of the upper flap as possible and on no account should the lower flap be dissected off this fascia. The upper flap is now inclosed in warm, dry, sterile lint and secured far back with tissue forceps. The lower flap being retracted on the fascia is treated in a similar manner and the operation for which the incision was made proceeds in the usual manner. If the operation is prolonged the warm, dry lint should be renewed.

The next step is the important one of adjustment. Upon the question of tension depends the necessity of a subcutaneous suture. I seldom use a suture but when I do it is as follows. I pass a lance-shaped slightly curved needle threaded with fine horsehair through the skin upon the slant at a little distance from one extremity of the wound; it then passes through the base of the upper and the apex of the lower flap continuously, its point of exit being through the skin on the slant at the other extremity of the wound. The suture is not tied and is removed at the end of 48 hours. Previously to inserting a suture, in all cases the following very important manipulation must be carried out. The object is to stretch the delicate apex of the upper flap, the least force will tear it off. therefore if forceps are used great care must be exercised. I find the best plan is gently to press this edge between the finger and thumb when it will be found gradu. ally to yield; at the same time the lower flap must be stretched and placed in position with suitable forceps. The delicate edge is then carefully spread in its proper position, the accuracy of which is best tested with a good large magnifying glass. The subsequent object of dressing is to keep this edge, together with the rest of the flap, in accurate flat position, remembering that undue pressure may cause damage. Sometimes I secure the wound by placing across a few strips of China silk and collodion, at others by merely placing collodion on the curved or flat glass dressing. When the glass is pressed flat the exact position of the flap can be seen at a glance. I prefer glass to celluloid or mica because it is rigid, a matter of importance when applying pressure. In all probability excellent results will be obtained in a dozen other ways. I use glass because I am accustomed now to its manipulation, but I do not propose to go into any further details on this point at present. I published a paper ${ }^{1}$ a little time ago giving some details, but I find that surgeons consider it is too much trouble, although they admit that the principle is correct.

With regard to pressure, it should be slight for the first few days, then gradually increased. Whichever rigid dressing is placed over the wound it should be covered by a large pad of wool. If no rigid dressing is thus used in all probability a contracted condition of the flap will result. Sooner than allow a wound to heal in bad position $I$ would always break it down and begin again.
At the present time I am more concerned with the artistic "effect produced when operating upon the more exposed parts of the body and particularly in those classes of scciety to which any undue blemish is of importance, especially in removing glands from the neck. The mechanical advantage of the operation in general I am well aware of. It is to be feared that only in these cases will the best results be obtained. We are all aware that wounds on the face heal rapidly but do they not invariaty leave ugly scars? I prefer to deal with these cases by converting the accidental cut to one on the slant at or as near the time of accident as possible and I have no hesilation in breaking a recently healed wound for this purpose. In such cases where the operation has been performed by a consulting surgeon the general practitioner must be called in to plap a most important part; not only should he personally be intrusted with the subsequent massa re but also with the general supervision for a considerable period and this should in all cases be explained to the patien before operation. These results would seldom be obtained with the poor who would not be found to present themselves once the wound was healed. The difficulties of the operation are not many but they will be met with-a very thin skin over a tense cyst or absce-s, and, on the contrary, a very lax condition of skin may tax the ingenuity of the beginner but not the experienced surgeon.

The very important question now to be considered is the one of massage. The massage of wounds and scars practically is not recognised and as a general rule may be said to be entirely neglected and yet is there any reason why it should not be carried out with as excellent result as in the case of bones, ligaments, muscles, \&c.? The moment a wound has healed over, as a rule, it is left to its fate and only when the skin has formed firm adhesions and contractions is the damage realised. In any ordinary wound involving the whole thickness of the skin the scar may be prevented from forming adhesions; it may be stretched, sof teved, and in many other ways improved.

In the case of my incision upon the slant there is perhaps only a very small amount of scar tissue, but though unseen it is there. Proper massage and stretching if necessary will promote circulation, restore contour, and prevent subcutaneous adhesions. Few patients would submit themselves continuously to the consultant or general practitioner after all apparent reason had gone for so doing and therefore $I$ am drawn to the conclusion that it is only by the coöperation of both that the best results will be obtained.

Wimpole-street, $W$.

\section{BRONCHO-PNEUMONIA AND PY ÆMIA.}

\section{BY IVY MCKENZIE, M.A, B.SC,' M.B., CH.B. GLASG.,} CARNEGIE RESEARCH SCHOLAR, PATHOLOGICAL DEPARTMENT, WESTERN INFIRMARY, AND UNIVERSITY OF GLASGOW ; LATE RESLDENT ASSISTANT PHYSICIAN, RUCHILL FEYER HOSPITAL, GLASGOW.

THE serious complications of measles are those which are associated with, or follow on, the initial catarrh of the respiratory passages, and of the complications the most serious is broncho-pneumonia. This condition may develop during the acme of the exanthem or it may not appear until the eruption has faded and the initial fever has passed off. The clinical symptoms and signs and the pathological appearances vary greatly; the disease may terminate fatally after two or three days or it may continue for three or four months and end in recovery. In some cases the symptoms of respiratory distress may be very alarming and the objective pulmonary signs very indetinite, while microscopic examination ray ve necessary to determine the nature of the lesion. In other cases respiratory difficulty may be absent and fever may never exceed $99^{\circ}$ or $100^{\circ} \mathrm{F}$. and for the most part be below $98.4^{\circ}$, while physical examination reveals the presence of extensive consolidation, and on postmortem examination there may be found advanced lobular consolidation with acute vesicular emphysema. No less varied is the number of pathogenic organisms that have been found in the bronchial secretion and in the inflammatory exudate. These include Fraenkel's pneumococci, Friedlander's bacilli, staphylococci, streptococci, pseudo-diphtberitic bacilli, and bacilli coli. It is believed that the pneumo coccus is present in the great majority of cases and it 
has been found by me in pure culture and associated with other organisms in 26 out of 34 cases examined. Not infrequently organisms may be found in the blood. In the examination of 12 cases, eight before death and four after death, in four of those examined before death organisms were found (two pneumococci, one staphylococci, and one streptococci), while in three out of the four examined after death organisms were found (one pneumococci, one staphylococci, and one bacilli coli). A case has recently been observed which constitutes a type altogether different from any of the many varieties usually seen and described. In this case, very acute in its course, the constitutional and nervous symptoms overshadowed the respiratory symptoms. The ultimate examination showed it to be a case of bronchopneumonia giving rise to pyæmia and solely due to the staphylococcus aureus. It is, moreover, the only case in a series of 34 examined in which the staphylococcus aureus was present unassociated with any other organism. The case presents features of a singular character, interesting from both a clinical and pathological standpoint. It is the only case of its kind noted in the clinical observation of 1000 cases of measles and in the pathological examination of 130 cases of broncho-pneumonia.

A boy, aged one year and eight months, came under observation on Sept. 26th, 1905. He was quite well until the $23 \mathrm{rd}$ when he took a "cold in the head." The eyes and nose discharged, cough was frequent, and he was not disposed to take his food. These symptoms increased in severity and were subsequently accompanied by considerable restlessness, until on the morning of the 26th a rash was noticed on the face. On the evening of the same day he was admitted to a measles ward in hospital. With regard to his previous health the child was said to have been healthy till the onset of the present illness. As to his family history and social conditions, the father and mother were alive and well. The patient was one of a family of four, all of whom were in good health. There was no evidence of constitutional disease in the history. He lived with his parents and three elder brothers in a house consisting of a room and kitchen, which was said to be well lighted and ventilated. He had always had plenty of nourishing food.

On admission (on the 26th) he was restless and slightly delirious. He was well nourished and developed. There was a profuse morbilliform rash all over the body. The conjunctivæ were suffused. the face was swollen and rhinitis was present. The tongue was cuated with a brown fur and Koplik's spots were present on the buccal mucosa. The throat was cor gested but there was no ulceration. The chest on inspection was seen to move freely and equably; the respirations numbered 52 per minute but were not laboured. On percussion the note over the pul. monary area was resonant throughout and on auscultation the respiratory murmur was everywhere accompanied by a moist inspiratory rî̀le and an occasional wheezing râle ; there was no evidence of consolidation. Cough was infrequent but secretion was not ejected. The area of cardiac dulness was marked on the right by the left sternal border, on the left by a line a quarter of an inch inside the nipple line and above by the third rib. The apex impulse was in the fourth interspace and a quarter of an inch inside the nipple line; the heart sounds were distinct, of good tone, and free from murmur. The pulse was regular in force and rhythm, of fairly good quality, and numbered 148 per minute. The abdomen was flaccid and the liver could be felt projecting beneath the right costal margin but the spleen could not be detected. The pupils were equal and reacted normally. The temperature was $101 \cdot 2^{\circ} \mathbf{F}$. On the 29 th the pro gress of the patient was not satisfactory. Although the measles rash had disappeared there was no abatement of the fever and contitutional symptoms. The suffusion had disappeared from the conjunctivæ but the eyes had a clear, bright, and glassy appearance which was unnatural. There was still a considerable amount of restlessness and during the previous night (the $28 \mathrm{th}$ ) he slept very little. The restlessness, moreover, was accompanied by a muttering delirium and while there was general nervous activity there appeared to be no cognisance of, or voluntary response to, surrounding influences. At times there was rolling of the eyeballs; the pupils were equal, moderately dilated, and reacted normally; the fundi oculorum appeared to be normal. There was no direct evirlence pointirg to a meningeal affection. Examination of the bones revealed nothing to account for the reslless and delirium. 'The temperature had not been high since armission; only on one occasion, when it registered $103.2^{\circ}$, had it risen above $102^{\circ}$. The colour was said to vary from time to time ; at one time he was flushed and at another pale and blanched. The respirations, while they did not vary much in rhythm, were at times somewhat laboured. The chest moved freely and equably on respiration; the percussion note was still normal throughout; at the left base and extending up to the angle of the left scapula and into the left axillary region there was an area over which the inspiratory part of the respiratory murmur was accompanied by an articulate crepitant riile. At no part, however, could the breathing be said to lave a tubular quality. Pleural friction was distinct in the left axillary region. The upper part of the left lung and the whole of the right lung were free from signs which would indicate serious complication. The condition of the heart had not changed. The pulse was regular but soft and easily compressed and numbered 148. The tongue was parched and coated with a brown fur. The throat was still slightly congested, The bowels moved regularly and the stools appeared to be normal. The patient took his nourishment willingly.

On Oct. 1st the child's condition had become progressively worse. Delirium was still present, the restlessness was extreme, and the keen glassy appearance of the eyes at once attracted attention. The temperature was not high, ranging a short distance on either side of $102^{\circ}$. The colour was pale with occasional flushings. The respirations varied from 50 to 60 per minute; the evidence of pulmonary complication was now unequivocal; all over the left base the percussion note was dull and resistant and the respiratory murmur was replaced by extremely tubular and superficial breath sounds. An exploratory puncture was negative. All over the rest of the chest there were occasional moist and wheezing rîles accompanying the respiratory murmur, but at no other part was consolidation detected. The area of cardiac dulness bad not changed. The first sound was weak and somewhat indefinite in tone but the second sound was distinct and emphatic. The pulse was regular in force and rhythm but easily compressible and numbered 140 per minute. The patient was having ten minims of a 10 per cent. solution of camphor every four hours. He took his nourishment of milk and water willingly; the stools were loose and very offensive and contained undigested material; he was rapidly losing flesh and a diet of raw meat juice and whey had been resorted to. On the 3rd any change that had taken place had been for the worse; tnere had been progressive emaciation and the patient was now very weak. The delirium was still present but the restlessness was modified; the eyes bad still their bright, sharp appearance; the pupils were equal and reacted normally and the fundi oculorum were normal. The tongue was dry and coated. The patient did not take his nourishment willingly and there had been occasional vomiting. The stools were very offensive and loose. A specimen of the urine was acid in reaction, contained a trace of albumin, but no blood or tube casts. The physical signs in the chest pointed to a rapidly advancing process. The whole basal region of the left lung was quite dull to percussion and the breathing in this situation was exquisitely tubular. Exploratory puncture again gave negative evidence. On the right side of the chest a resonant note was found throughout, although there was a relative increase in the resistance to the percussing finger, as examination was carried from the axillary region to the vertebral column. At the angle of the right scapula the respiratory murmur was replaced by breathing of a tubular quality and crepitant râle was abundant; these signs of consolidation were present all over the posterior aspect of the right lung, but diminished in amount in proportion to the distance from the inferior angle of the scauula. In the right axillary region pleural friction was heard. The area of cardiac dulness extended on the right to the mid-sternal line and on the left to the nipple line and upwards to the third rib. The first sound was toneless and at times the second sound was indistinct. The pulse was regular in rhythm but irregular in force and was very feeble even at the acme of its strength. It numbered 180 per minute. The patient died at 2 A.M. on the morning of the 4th.

Summary of olinioal history.-A child, aged one year and eight months, strong and well nourished and with a history of good bealth, was found suftering from an attack of measles associated with delirium. The rash appeared on the fourth day of 1llness and on that date, except for the delirium, there was nothing exceptional about the case. On 
the sixth and seventh days of illness the rash had almost gone, but the temperature had not settled and the child was very restless and delirious. Evidence of commencing pulmonary consolidation could be detected at the left base but the nervous symptoms were not such as one might expect at the beginning of an uncomplicated pneumonia with moderate temperature $\left(102^{\circ}\right)$. Examination of the nervous system and of the bones afforded no evidence of the presence of meningitis or osteomyelitis. On the ninth day of illness delirium was still present and consolidation at the left base was so advanced as to suggest the presence of fluid. The patient died on the eleventh day from the onset of the measles symptoms and on the eighth day from the appearance of the rash and on the fifth day from the detection of the broncho-pneumonic signs. The most prominent features of the case were the persistent delirium with moderate temperature and the very rapid and advanced consolidation of the left pulmonary base.

Necropsy. - At the post-mortem examination the body was found to be well developed but poorly nourished, subcutaneous fat having almost entirely disappeared. The muscles were pale. There was slight congestion of the tonsils and pharynx but no evidence of ulceration. The mucous membrane of the larynx and trachea was greyish red, contained several ecchymosed patches, and was covered at parts with thin purulent secretion. There was no fluid in either pleural cavity. The left lung weighed eight and a quarter ounces; from its bronchus there exuded a quantity of thick creamy pus. The upper lobe with the exception of the atelectatic lingula was crepitant throughout, while the lower lobe was firm, cedematous, and non-crepitant. The upper lobe weighed one and three-quarter ounces, while the lower lobe weighed six and a half ounces and sank readily in water. The upper lobe had a pale greyish appearance except the lingula which was bluish-red. The lower lobe was dark red in appearance, presented numerous minute sub-pleural hæmorrhages, and was covered in its posterior aspect with a thin fibrinous exude. On section the upper lobe, except for the lingula, was inflated throughout its extent. A small quantity of thin purulent secretion was present in the bronchioles. The lower lobe was quite hepatised and from the cut surface there exuded a bloody and serous exudate, while thick creamy pus could be expressed from the bronchial apertures. The right lung weighed four and a quarter ounces and floated in water. A thick muco-purulent secretion could be seen in the bronchus. On its posterior aspect there was a layer of fibrinous exudate and posteriorly the organ had a brownish-red appearance with areæ of lobular collapse, while anteriorly the lung was grey in appearance and crepitant throughout. On section, a narrow strip of consolidation bordered the posterior aspect of the lung, varying in thickness from oneeighth of an inch to half an inch, and thickest at the centre of the posterior aspect of the lower lobe. From the bronchi there could be expressed drops of creamy purulent material ; the bronchial mucous membrane was deeply injected. The bronchial glands were large, pale, and soft. The pericardial sac contained about one and a half ounces of straw-coloured fluid. The heart was contracted; at the extreme left of the base was a small area, about half a square inch in extent rough and coated with a fibrinous exudate. The left ventricle was empty, the left auricle contained some partly coagulated blood, and in the right chambers were large white moist fibrin clots. Close examination of the mural endocardium revealed the presence of small yellowish specks which were about one millimetre in diameter (Fig. 2). The valves and great vessels appeared to be normal. The cardiac muscle was pale and soft. The abdominal cavity was devoid of fluid. The liver weighed 16 onnces; it was brownish-red in colour, with scattered pale areæ of about a quarter of a square inch in extent. Very minute yellowish specks from one millimetre to two millimetres in diameter were seen on the surface. The hepatic tissue was slightly congested and the gall-bladder was distended. The spleen weighed one and a quarter ounces. It was somewhat congested, of firm consistence, and the follicles were distinctly swollen. On the surface were seen minute yellowish specks, as in the liver and heart. The kidneys each weighed one and three quarter ounces. They were pale in appearance and the capsule came off easily. On the surface could be seen small yellow specks, rather larger than those in the liver and spleen, and section showed them to be minute abscesses. The cortex was yellowish-brown and the pyramids were pale. The mesenteric glands were pale, swollen, and soft. The stomach contained some greenish fluid and the mucous membrane, which was pale, was covered in parts with bile-stained slimy mucus. The skull appeared to be normal. The sinuses of the dura mater and the veins of the pia mater were distended with blood. The convolutions appeared to be distinctly flattened. The brain substance was soft, pulpy, odematous, and anæmic, only the larger vessels containing blood. The ventricles were moderately distended and contained each about two ounces of clear serous fluid.

Bacteriological examination.-1. Film examination. Examination of smears from the bronchial exudate, pneumonic exudate, and spleen pulp showed the presence of Gramstaining micrococci, apparently staphylococci. 2. Cultural examination. Tubes of bouillon inoculated from the pneumonic exudate, spleen pulp, pericardial fluid, and cerebro spinal fluid, and grown at $37^{\circ} \mathrm{C}$, , showed at the end of 24 hours growths of staphylococci. Separation cultures were made from the bouillon on to agar slopes and Petri's capsules and these presented colonies of staphylococcus aureus only. 3. Inoculation. From a fresh sub.culture of the growth in bouillon intra-peritoneal inoculation of a mouse caused death in 18 hours from septicæmia due entirely to staphylococcus aureus, and subcutaneous inoculation resulted in abscess formation with recovery.

Histological examination - Histological examination of the tissue of the left lower pulmonary lobe pointed to an advanced inflammatory condition, commencing in the bronchioles. The smaller bronchi and bronchioles were filled with purulent and mucous material, together with desquamated epithelium and a few blood cells; the peribronchial capillaries and arterioles were very much dilated. (Fig. 1.) Some of the alveoli in the neighbourhood of the

\section{FIG. 1.}

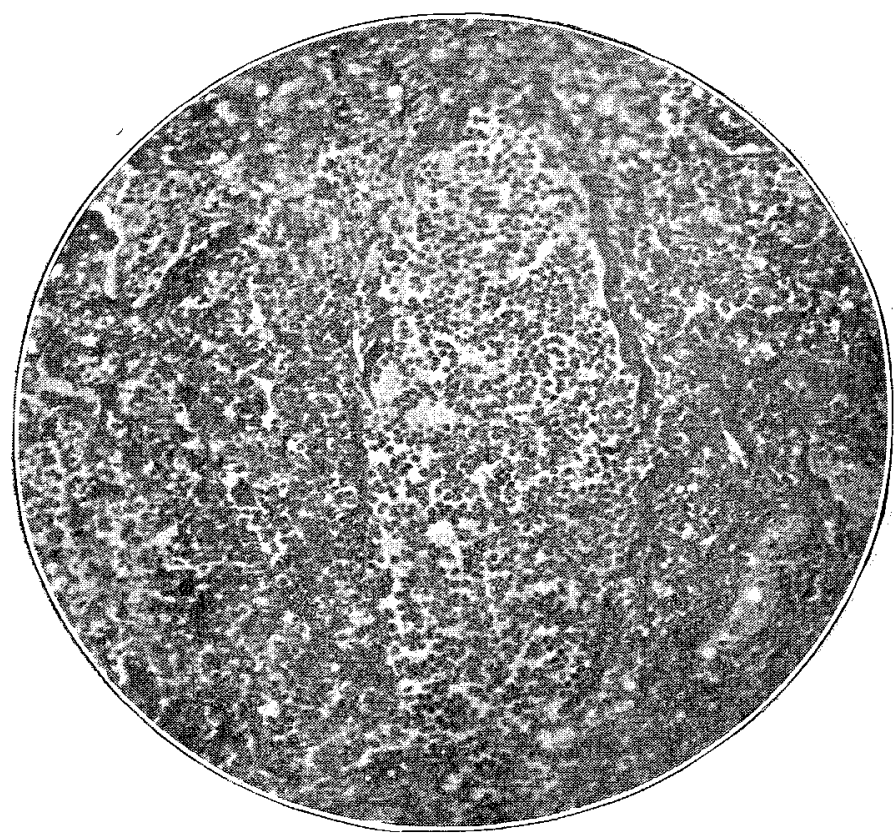

Bronchiole filled with purulent exudation. The surrounding pulmonary tissue is infiltrated and the peri-bronchial capillaries are dilated, $\times 100$. Stain, hæmatoxylin and eosin.

bronchioles showed the presence of a fibrinous exudate. The lung parenchyma, which appeared to be affected throughout, presented two kinds of changes: 1. Area in which there was dense purulent infiltration of alveoli, more especially in the neighbourhood of bronchioles. In addition to the numerous pus cells there were degenerate epithelial cells, a very few blood cells, and granular débris; the capillaries and arterioles were distended. 2. Area in which the changes were not so advanced; here the alveoli were small, the epithelium was swollen, with indistinct nuclei ; there were numerous de-quamated epithelial cells and granular débris a few polymoiphs and tree blood cells were also present; the capillaries and arterioles were distended; the changes in these areæ corresponded exactly to the description given by Aufrecht of what he terms "alveolar atelectasis." GramWeigert stained sections showed, in addition to the fibrinous exudation in the alveoli near the bronchi, isolated cocci in the purulent contents of the treoli and bronchi, and also 
groups of cocci in the arterioles (bronchial) and capillaries. Examination of the heart, liver, spleen, and kidneys showed, in addition to the cloudy swelling usually seen in acute febrile conditions, the presence of a large number of minute abscesses varying in size from one millimetre to two millimetres in diameter. Gram-Weigert stained sections showed cocci in these abscesses and, in addition, minute arterioles and capillaries plugged with staphylococci. The condition in the heart migbt be taken as representative of what is seen in the other organs. Fig. 2 shows a small abscess beneath

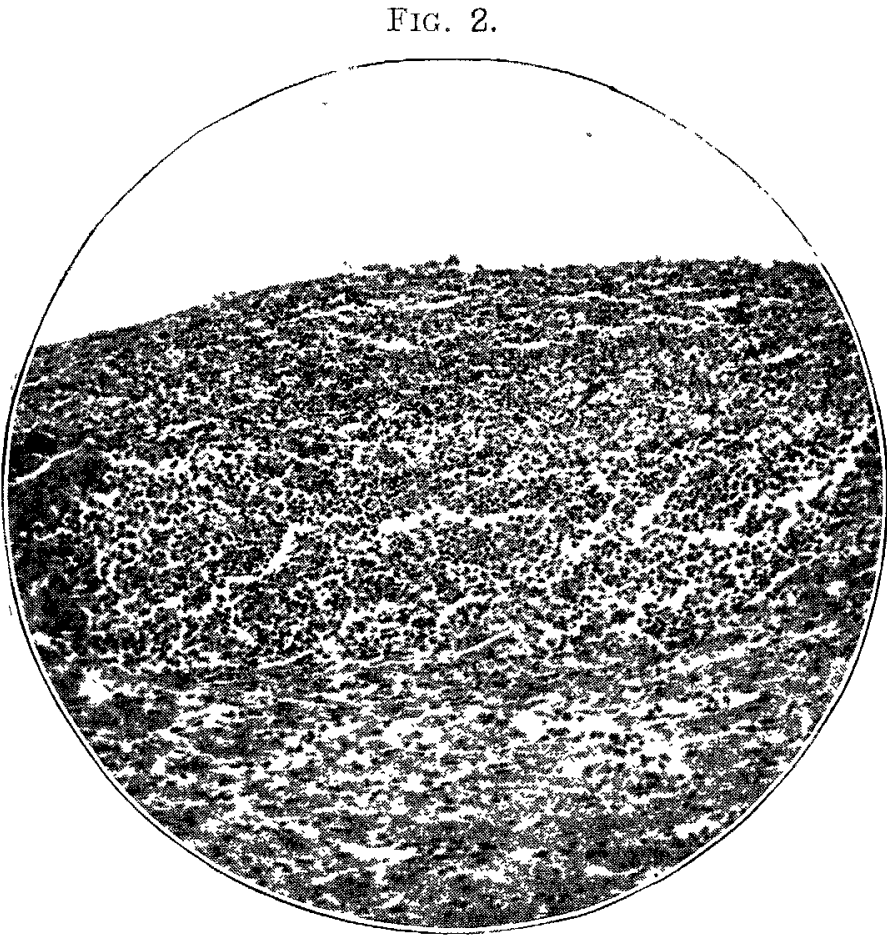

Small abscess beneath the endocardium, showing a limited purulent exudation with the remnants of a few muscle fibres. $\times 100$. Stain, hæmatoxylin and eosin.

the endocardium and Fig. 3 a capillary loop plugged with staphylococci.

Conclusions.-The main interest of the case arises from the relation of the symptoms to the pathological findings. While in measles, especially in young children, a certain amount of nervous disturbance not infrequently accompanies a high temperature, with the decadence of the fever the

FIG. 3.

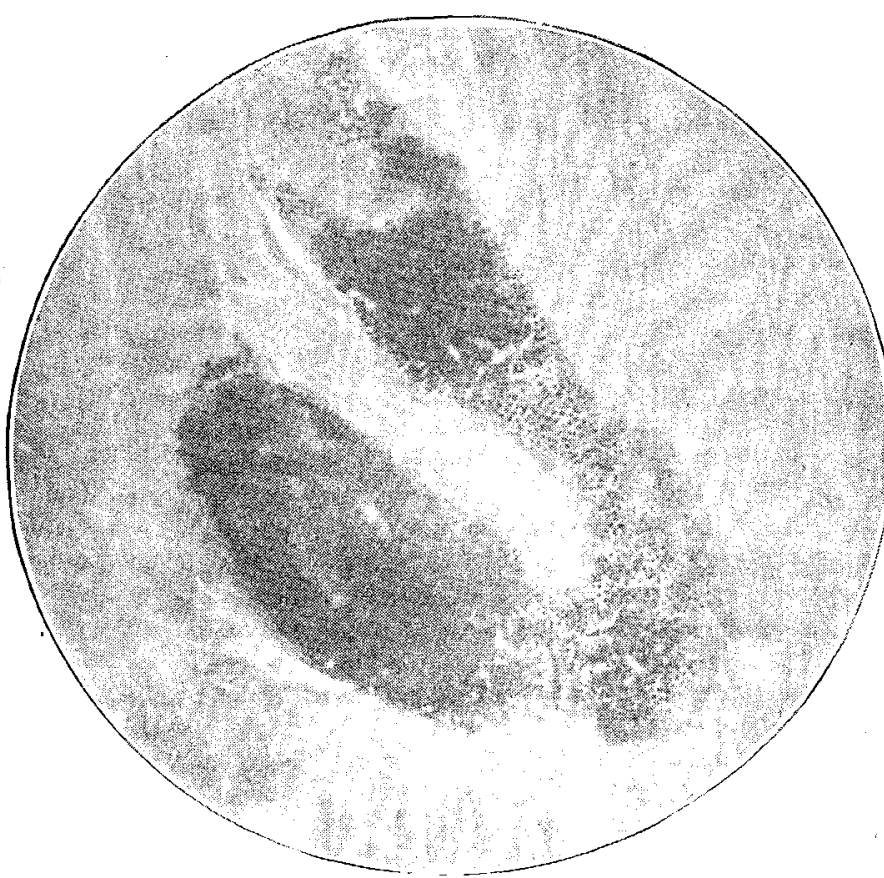

Capillary loop beneath the enclocardium in the muscular tissue, plugged with staphylococci. $\times 1000$. Stain, Gram.

nervous symptoms always disappear. A true meningitis has not been recorded as complicating the onset of measles. In broncho-pneumonia, on the other hand, children often show signs of nervous disturbance; a commencing pneumonia is not infrequently associated with an appearance of nervous depression, the child being dull, languid, and apathetic. while in other cases there is a considerable restlessness, It is, however, in advanced and protracted cases that evidence of cerebral disturbance is most frequently seen; in such cases there is incessant restlessness, sometimes convulsions, and subsequent rigidity or paresis of muscles. These cases are usually fatal and on post-mortem examination no abnormality of the brain or meninges can be detected microscopically. In other cases, again, a pneumococcic meningitis with the usual signs and symptoms may complicate the broncho-pneumonia.

The case under consideration presented evidence of nervous disturbance which, while it suggested meningitis, resembled more the extreme restlessness and delirium usually associated with acute osteomyelitis, acute phlegmonous cellulitis, or a very severe scarlet fever intoxication. The pathological examination, in view of the clinical symptoms, suggests the idea that there occurred in the lungs a severe inflammation the.constitutional clinical manifestations of which resembled those of acute inflammation of bone or cellular tissue. The history of the case may be considered as .(1) an inflammation due to the presence of a virulent form of staphylococcus aureus, beginning in the smaller bronchi and giving rise to the signs of a pulmonary consolidation; (2) the rapid progress and profuse infiltration and exudation giving rise to complete consolidation and atelectasis of the left lower lobe; and (3) the exalted virulence of the organism producing such a growth in the blood as to give rise to embolic infections of the various internal organs, leading to small abscess formations; and (4) the whole process resulting from infection by a highly virulent organism, the constitutional symptoms of intoxication overshadowed the effects of the local pulmonary lesion.

I am indebted to Dr. A. Johnston, physician superintendent of the Ruchill Fever Hospital, Glasgow, for permission to publish this case.

\section{Olinital a hotes:}

\section{MEDICAL, SURGICAL, OBSTETRICAL, AND THERAPEUTICAL.}

\section{A CASE OF TUBERCULOSIS PULMONALIS WITH SOME SYMPTOMS OF MENINGITIS; RECOVERY}

By Willitam Bertram Hill, M.B., Ch.B. Vict., RESIDENT MEDICAI OFFICER TO THE LEEDS SAMATORIUM FOR CONSUMPTIVES.

THE patient was a man, aged 26 years, who was admitted into the Leeds Sanatorium for Consumptives on Oct. 3rd, 1905, suffering from pulmonary tuberculosis which involved the upper and middle lobes of the right lung. On admission he weighed 9 stones $11 \frac{1}{2}$ pounds and measured 5 feet $10 \frac{1}{2}$ inches in height. His temperature at 4 P.M. was $99 \cdot 2^{\circ} \mathrm{F}$; his pulse and respirations were respectively 78 and 20 per minute. His sputum measured about a quarter of an ounce daily ; tubercle bacilli were present. There was marked dulness over the upper and middle lobes with bronchial breathing and whispering pectoriloquy; there were no râles. He was ordered to spend the whole day in the open air and his exercise was limited to one mile. He remained in this condition until Oct. 22nd, on which day his temperature varied from $100 \cdot 2^{\circ}$ to $101^{\circ}$; his pulse and respirations were respectively 80 and 18 per minute. His bowels were constipated. He complained of slight headache and vomited twice after food. On the $23 \mathrm{rd}$ his temperature varied from $99.2^{\circ}$ to $102 \cdot 8^{\circ}$; his pulse and respirations were respectively 80 and 18 per minute; his bowels were confined; his headache was more severe. On the 24th his temperature was still higher, reaching $105^{\circ}$ at 4 P.M. The pain in the head was much increased; it was vertical in character, continuous, and at times was so acute that he cried out. He vomited after taking soda water and milk. His pulse was 80 per minute, full and bounding in character. At time she was slightly delirious. On the 25 th it was noticed that there was an internal strabismus of the right eye and that the pupils were contracted, but reacted to light and accommodation. The patient himself complained that he did not seem to see 